\title{
Moderation effects of food intake on the relationship between urinary microbiota and urinary interleukin-8 in female type 2 diabetic patients
}

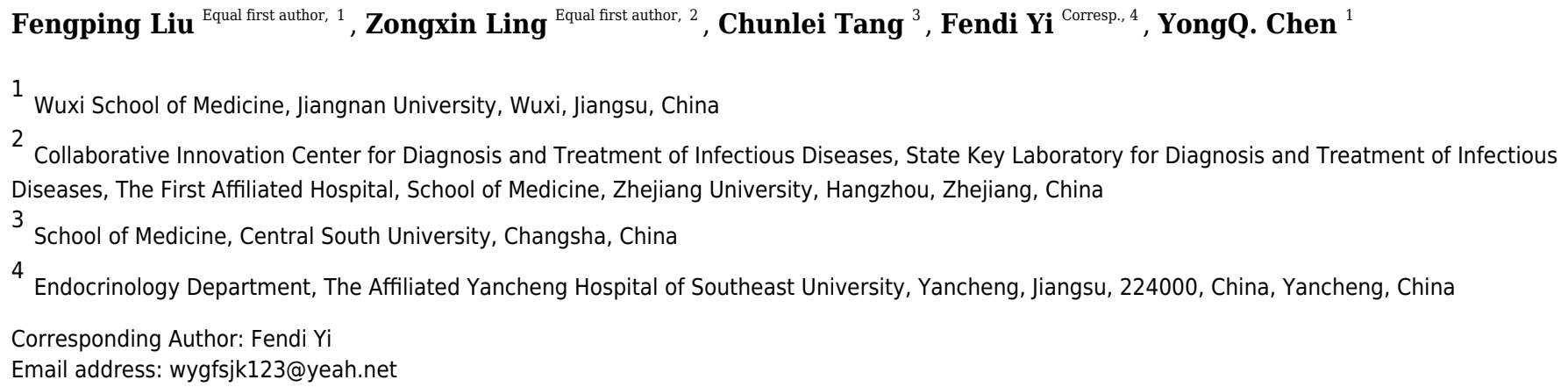

Background. Our previous study demonstrated that the composition of the urinary microbiota in female patients with type 2 diabetes mellitus (T2DM) was correlated with the concentration of urinary interleukin (IL)-8. As the composition of urine is mainly determined by diet, diet might mediate the correlation. Methods. Seventy female T2DM patients and 70 healthy controls (HCs) were recruited. Midstream urine was used for the urine specimens. Urinary IL-8 was determined by enzyme-linked immunosorbent assay. A Chinese Food Frequency Questionnaire was used to collect food intake data. The independent variables in the hierarchical regression analysis were the relative abundances of the bacterial genera and species that were significantly different between the T2DM and HCs and between the T2DM patients with and without detectable urinary IL-8, and the bacterial genera associated with IL-8 concentration in the multiple regression model reported in our previous research. IL-8 concentration was the dependent variable, and nutrient intakes were moderator variables. Results. Fiber and vitamin B3 and E intake exerted enhancing effects, and water intake exerted a buffering effect, on the positive relationship between the relative abundance of Ruminococcus and IL-8 concentration ( $p<$ $0.05)$. Cholesterol and magnesium intake exerted enhancing effects on the positive relationship between the relative abundance of Comamonas and IL-8 concentration ( $p<$ 0.05). Conclusion. Modulating T2DM patients' dietary patterns may prevent bladder inflammation. 
1 Moderation effects of food intake on the relationship between urinary microbiota and urinary 2 interleukin-8 in female type 2 diabetic patients

3 Fengping Liu $^{1 *}$, Zongxin Ling ${ }^{2 *}$, Chunlei Tang ${ }^{3 *}$, Fendi Yi ${ }^{4 \#}$, YongQ. Chen ${ }^{1 \#}$

$4{ }^{1}$ Wuxi School of Medicine, Jiangnan University, Wuxi, Jiangsu, 214122, China

$5{ }^{2}$ Collaborative Innovation Center for Diagnosis and Treatment of Infectious Diseases, State Key

6 Laboratory for Diagnosis and Treatment of Infectious Diseases, The First Affiliated Hospital, School of

7 Medicine, Zhejiang University, Hangzhou, Zhejiang, 310003, China

$8{ }^{3}$ School of Medicine, Central South University, Changsha, 41000, China

$9{ }^{4}$ Endocrinology Department, The Affiliated Yancheng Hospital of Southeast University, Yancheng, 10 Jiangsu, 224000, China

$11{ }^{*}$ These authors contributed equally to this work

13 Corresponding Author:

14 Prof. Fendi Yi

1575 Juchang Road, Yancheng, Jiangsu, 224000, China

16 E-mail: wygfsjk123@yeah.net; Tel: +86-151-8920-0355; Fax: +86-515-81608521

17 Prof. Yong Q. Chen

181800 Lihudadao Road, Wuxi, Jiangsu, 214122, China

19 Email: yqchen@jiangnan.edu.cn; Tel: +86-510-8591-3906; Fax: +86-510-8592-8605 


\section{Abstract}

36 Background. Our previous study demonstrated that the composition of the urinary microbiota in female patients with type 2 diabetes mellitus (T2DM) was correlated with the concentration of urinary interleukin (IL)-8. As the composition of urine is mainly determined by diet, diet might mediate the correlation. Methods. Seventy female T2DM patients and 70 healthy controls (HCs) were recruited. Midstream urine was used for the urine specimens. Urinary IL-8 was determined by enzyme-linked immunosorbent assay. A Chinese Food Frequency Questionnaire was used to collect food intake data. The independent variables in the hierarchical regression analysis were the relative abundances of the bacterial genera and species that were significantly different between the T2DM and HCs and between the T2DM patients with and without detectable urinary IL-8, and the bacterial genera associated with IL-8 concentration in the multiple regression model reported in our previous research. IL-8 concentration was the dependent variable, and nutrient intakes were moderator variables.

Results. Fiber and vitamin B3 and E intake exerted enhancing effects, and water intake exerted a buffering effect, on the positive relationship between the relative abundance of Ruminococcus and IL-8 concentration $(\mathrm{p}<0.05)$. Cholesterol and magnesium intake exerted enhancing effects on the positive relationship between the relative abundance of Comamonas and IL-8 concentration $(\mathrm{p}<0.05)$.

Diabetes mellitus (DM) is a severe chronic disease that is recognized as a global public health problem. In recent decades, the prevalence of DM has increased substantially. Globally, about 1 in 11 adults have DM (90\% have type 2 diabetes mellitus (T2DM)) (Zheng et al., 2018). Infections were more common prevalent with T2DM than among respondents without diabetes (Carey et al., 2018), especially urinary tract infection (UTI) (Wilke et al., 2015). UTI is of major concern, and many studies have demonstrated an increased prevalence of UTI in T2DM patients (Wilke et al., 2015; Hirji et al., 2012; Nichols et al., 2017), especially in female patients (Hirji et al., 2012). Traditionally, UTI has been thought to be caused by bacterial invasion of the patient's bladder. However, studies on the urinary microbiota have caused this concept to be questioned over the past decade, as DNA sequencing and expanded quantitative urine culture technique have shown that bacteria live in almost all individuals' bladders, both UTI patients and healthy subjects (Price et al., 2016; Thomas-White et al., 2018). Therefore, the presence of bacteria in the bladder of UTI patients may just indicate an altered bacterial profile compared to that of healthy individuals rather than the invasion of new bacteria.

An increasing body of evidence suggests that T2DM is associated with profound gut dysbiosis (Tai et al., 2015; Wang et al., 2017). Food intake patterns can modulate the dysbiosis, which in turn impacts metabolic health (David et al., 2014; Simpson \& Campbell, 2015). The kidneys are primary excretory 
69

organs, and are responsible for the elimination of waste metabolites. Food intake patterns determine the metabolites in urine, which may affect the bacterial profile in the bladder (Shields-Cutler et al., 2015).

It has been reported that interleukin (IL)-8 can function as a diagnostic biomarker for UTI, with a sensitivity of $93 \%$ and a specificity of $90 \%$, which is much greater than the sensitivity and specificity of urine culturing (Rao et al., 2001). Similarly, another study demonstrated that urinary IL-8 could be considered as a surrogate marker for the rapid diagnosis of bacteriuria (Zaki, 2008). Therefore, we detected the urinary IL-8 concentration in our previous research, and found that 46 out of 70 subjects had detectable concentrations of urinary IL-8 (Ling et al., 2017).

Our previous research demonstrated that T2DM patients had lower bacterial diversity in urinary microbiome than healthy controls (HCs). There were significant differences in the relative abundance of multiple urinary bacterial genera between T2DM patients and HCs, including Lactobacillus, Prevotella, and Pseudomonas (Liu et al., 2017). Subgroup analysis showed that T2DM patients with detectable concentrations of urinary IL-8 (WIL8) had a different urinary bacterial profile from those with no detectable concentrations of urinary IL-8 (NIL8); indeed, the WIL8 group had notable elevations and reductions in several bacterial genera and species compared to the NIL8 group. Moreover, subjects in the T2DM group with higher or lower relative abundance of specific urinary bacteria genera, relative to HCs, had different concentrations of IL-8. Additionally, several urinary genera were significantly associated with the urinary IL-8 concentration in T2DM patients, including Ruminococcus, Anaerotruncus, and Lactobacillus. Moreover, the WIL8 group had significantly higher urinary concentrations of nitrites and leukocyte esterase than the NIL8 group (Ling et al., 2017).

As stated above, the urinary microbiome profile in T2DM females is different from healthy controls, and the bacterial dysbiois is related to the expression level of IL-8. Studies on gut microbiome have shown that diet regulate the composition of microbiome and inflammation (Tilg \& Moschen, 2015). On one hand, gut microbiome can rapidly respond to altered diet. Walker AW et al. demonstrated that increassed intake of resistant starch can substantially alter the species composition of the colonic microbiome (Walker et al., 2011). David LA et al. reported that short-term consumption of diets composed entirely of animal or plant products resulted in microbial community structure (David et al., 2014). On the other hand, studies have shown that intestinal dysbiosis led to an abnormal adaptive immune response that increased inflammatory gut diseases (Seksik et al., 2003). However, to date, no studies have shown whether the relationship between urinary microbiome and IL-8 level is regulated by nutrients intake. Therefore, our present study aimed to analyze the regulating role of nutrient intake on the correlation between the urinary microbiome and IL-8 using moderation effect model. If nutrients intake has a moderating role, clinicians can precisely adjust patient's dietary composition based on the correlation between urinary microbiome and IL-8 concentration.

Peer) reviewing PDF | (2019:06:38336:2:0:NEW 17 Dec 2019) 
103

104

105

106

107

108

109

110

111

112

113

114

115

116

117

118

119

120

121

122

123

124

125

126

127

128

129

130

131

132

133

134

135

136

\section{Materials \& Methods}

\section{Recruitment of subjects}

Briefly, a case-control design was used in our previous study, in which female T2DM patients were individually matched to HCs according to age, marital status, and menstrual status. The same data were used in the present study. The participants were recruited from the First Affiliated Hospital, School of Medicine, Zhejiang University, from July 2, 2015 to January 2, 2016. For every 10 T2DM patients recruited, 10 healthy controls who matched the patients' age, marriage and menstrual status were recruited. Subjects with the following attributes were excluded: UTI in the previous month; use of antibiotics, probiotics, prebiotics, or synbiotics in the previous 3 months; inability to complete the questionnaire; menstruation; urinary incontinence; known anatomic urinary tract abnormalities (e.g., cystoceles, hydronephrosis, renal atrophy, or neurogenic bladder); or urinary catheter use. The Ethics Committee of the First Affiliated Hospital, School of Medicine, Zhejiang University, approved the study (ref. 295). Written informed consent was obtained from each patient before enrollment.

\section{Urine specimen collection, DNA isolation and sequencing, and IL-8 detection}

A self-designed midstream urine collection technique, which was reported in our previous study, was used to collect the first urine of the day (Liu et al., 2017). Bacterial DNA was isolated using magnetic beads, and the 16S rRNA gene V3-V4 regions of microbial genomic DNA were amplified by PCR (forward primer, 5'-ACTCCTACGGGAGGCAGCAG-3'; reverse primer, 5'GGACTACHVGGGTWTCTAAT-3'). Amplicons were then normalized, pooled, and sequenced using an Illumina MiSeq desktop sequencer $(2 \times 300$ bp paired-end run). Sequence read processing was performed using QIIME (version 1.9.0), which included quality trimming and demultiplexing. Operational taxonomic unit (OTU) selection, using Usearch (version 1.11.1), included dereplication (-derep_fullength), clustering (--cluster_fast, --id 0.97), and chimera detection (--uchime_ref). Taxonomic assignment for individual datasets was conducted using Greengene (version 13.8). The output file was further analyzed using Statistical Analysis of Metagenomic Profiles software (version 2.1.3). Urinary IL-8 concentrations were determined using enzyme-linked immunosorbent assay (ELISA) kits (RayBiotech, Inc., Norcross, GA, USA).

\section{Food intake assessment and nutrient intake calculations}

The influence of diet on the human microbiota is long term, so almost all previous studies on the relationship between the human microbiota and nutrition used the Food Frequency Questionnaire (FFQ) (Mandal et al., 2016; Ribeiro et al., 2017; Singh et al., 2017), which requires participants to recall food intake in the previous year. Therefore, a Chinese version of the FFQ was used to assess food intake (Zhao et al., 2010). Prior to the present study, we conducted a pre-experimental study. We evaluated the reproducibility of the FFQ (Chavarro et al., 2009), in which 10 volunteers were invited to filled the FFQ 
137 for twice, e. g. $\mathrm{FFQ}_{1}$ and $\mathrm{FFQ}_{2}$. The interval time between the two interviews was two weeks. FFQ 1 and $138 \mathrm{FFQ}_{2}$. Then we assessed the correlation coefficient of nutrients intake of energy, protein, fat and 139 carbohydrate, and found they were significantly correlated in $\mathrm{FFQ}_{1}$ and $\mathrm{FFQ}_{2}(p<0.05$; data was not 140 shown) indicating that the reproducibility was good. In the meantime, we conducted a qualitative 141 interview. Based on their suggestions, a face-to-face interview was carried out in each participant's 142 kitchen, where the sizes of the participant's dinnerware items were evaluated. The participants were asked 143 to recall their food and water intake patterns over the previous year (up to the day of urine sample 144 collection). Before the interviews, we visited local supermarkets to convert the quantities of certain foods 145 to weights, as most participants were able to remember the amount, but not the weight, of the food they 146 ate. Subsequently, we separated the elements of the reported food intake into large, moderate, and small 147 portion sizes. Data on the intake of Chinese herbs and supplements were also collected. Water intake 148 included drinking water and the water content of foods. The mean duration for assessing each patient's 149 food intake data was $90 \mathrm{~min}$. MATLAB software (version 7.0) was used to convert the amount of food

150

151

152

153

154

155

156

157

158

159

160

161

162

163

164

165

166

167

168

169

170 intake into daily nutrient intake.

\section{Statistical analysis}

Statistical analysis was performed using SPSS software (version 21.0). For continuous variables, independent $t$-tests were applied. For categorical variables, either Pearson's chi-square tests or Fisher's exact tests were used depending on assumption validity. A moderation effect can be enhancing, buffering or antagonistic (Fairchild \& MacKinnon, 2009). Therefore, hierarchical regression analysis was used to analyze the moderating effects of nutrient intake on the relationships between the urinary microbiota and IL-8 concentration. To establish hierarchical regression models that would be likely to be true representations of real relationships, the independent variables selected were the bacterial genera and species that exhibited differences in relative abundances between the T2DM and HC groups and between the WIL8 and NIL8 groups of T2DM patients, and also the bacterial genera that contributed to the urinary IL-8 concentration in the T2DM cohort (according to our previous research). The moderator variables were nutrient intakes; the dependent variable was urinary IL-8 concentration; and the control variables were fasting blood glucose, urine glucose, age, menstrual status, and body mass index (Liu et al., 2017; Ling et al., 2017). Before conducting hierarchical regression analysis, Pearson's correlation analysis was used to determine whether the independent and dependent variables were significantly correlated. The independent variables that were significantly correlated with the dependent variable were then entered into hierarchical regression models. The hierarchical regression analysis featured three-steps. First, we entered age, body mass index, fasting blood glucose, urine glucose, and menstrual status as control variables, as our previous study showed that these factors affected the urinary microbiota. Second, we entered other independent variables, such as the relative abundances of bacteria and nutrient intakes. 
171 Third, we entered the "bacteria $\times$ nutrient" interaction terms. The regression effects were then analyzed.

172 All tests of significance were two-sided, and $\mathrm{p}<0.05$ was considered significant. After obtaining $\beta$ values

173 for the control variables, main effects, and interaction effects, ModGraph-1 software was used to calculate

174 cell means to display moderation effects in a graphical manner. ModGraph-1 software calculated the

175 effects of high, medium, and low nutrient intake on the relationships between the relative abundance of

176 bacteria and IL-8 concentration.

\section{Results}

178 Patient characteristics, DNA sequencing, and IL-8

179 Seventy T2DM patients were recruited. Their characteristics are shown in Table 1. A total of $1803,981,519$ reads were obtained, accounting for $76.93 \%$ of the valid reads. The mean read length was 438 181 bp (range, 423-486 bp). Good's coverage estimator was 98.00\%. Sequence data from this study are 182 deposited in the GenBank Sequence Read Archive (accession no. SRP 087709). The mean urinary IL-8 183 concentration was 42.26 $\pm 64.66 \mathrm{pg} / \mathrm{mL}$ (Liu et al., 2017; Ling et al., 2017).

Several nutrients exhibited moderation effects on the associations between the urinary microbiota and urinary IL-8 concentrations

In our previous studies, the relative abundance of 33 bacterial genera-level and 9 bacterial specieslevel OTUs showed significant differences between T2DM patients and HCs (Figures S1 and S2), 21 bacterial genera-level and 10 bacterial species-level OTUs showed significant differences between the WIL8 and NIL8 T2DM subgroups (Figures S3 and S4), and 18 bacterial genera were predictors of urinary IL-8 in a stepwise regression analysis (Table S1). In addition, we identified T2DM patients with significant differences in their urinary microbiota compared with HCs, grouping them according to the bacterial genera present in their urine samples (" $\geq \mathrm{HCs}$ " or " $<\mathrm{HCs").} \mathrm{We} \mathrm{then} \mathrm{analyzed} \mathrm{the} \mathrm{urinary} \mathrm{IL-8}$ concentrations for the T2DM patients with significantly high or low abundance of a given genus, relative to the HCs. The IL-8 concentration was low in T2DM patients with high Acinetobacter, Microbacterium and Megamonas abundance, but high in T2DM patients with high Pseudomonas and Klebsiella abundance (Figure S5) (Liu et al., 2017; Ling et al., 2017). These bacterial genera were included in the hierarchical regression analysis (Table S2).

The relative abundances of Ruminococcus, Comamonas, Cytophaga, Providencia, Anaerotruncus, Giesbergeria, Meiothermus and Luteibacter were correlated with increased urinary IL-8 concentrations, whereas Cloacibacterium was correlated with decreased IL-8 (Table S3). When these bacteria were used as independent variables, urinary IL-8 was used as the dependent variable, and nutrient intake variables 202 were used as moderating variables (Table 2), hierarchical regression analysis showed that several 203 nutrients moderated the correlation between the urinary microbiota and IL-8. 
204 The relative abundance of Ruminococcus and water intake had significant effects on urinary IL-8 205 concentration (Table S4). The "Ruminococcus $\times$ Water" interaction effect was also significant, and it 206 explained $39.00 \%$ of the variance in IL-8 concentration. The strongest negative association between the 207 relative abundance of Ruminococcus and IL-8 concentration occurred in patients who reported a high 208 water intake, while the weakest negative association occurred for patients who reported a low water 209 intake (Figure 1A). This suggested that water intake buffered the relationship between the relative 210 abundance of Ruminococcus and IL-8 concentration.

211 The relative abundance of Ruminococcus and fiber intake had significant effects on urinary IL-8 212 concentration (Table S5). The "Ruminococcus $\times$ Fiber" interaction effect was also significant, and it 213 explained $41.70 \%$ of the variance in IL-8 concentration. In addition, the strongest positive association 214 between the relative abundance of Ruminococcus and IL-8 concentration occurred in patients who 215 reported a high fiber intake, whereas the weakest association occurred in those who reported a low fiber 216 intake (Figure 1B). This indicated that fiber intake enhanced the positive correlation between the relative abundance of Ruminococcus and IL-8 concentration.

In addition, the relative abundance of Ruminococcus and vitamin B3 intake had significant effects on urinary IL-8. The "Ruminococcus $\times$ Vitamin B3" interaction effect was also significant, and it explained $32.00 \%$ of the variance in IL-8 concentration (Table S6). The strongest positive association between the relative abundance of Ruminococcus and IL-8 concentration occurred in patients who reported a high vitamin B3 intake, whereas the weakest association occurred in patients who reported a low vitamin B3 intake. This indicated that vitamin B3 intake enhanced the positive correlation between the relative abundance of Ruminococcus and IL-8 concentration (Figure 1C).

The relative abundance of Ruminococcus and vitamin E intake also had significant effects on urinary IL-8. The "Ruminococcus $\times$ Vitamin E" interaction effect was also significant, and it explained 34.40\% of the variance in IL-8 concentration (Table S7). Surprisingly, the strongest positive association between the relative abundance of Ruminococcus and IL-8 concentration occurred in patients who reported a high vitamin $\mathrm{E}$ intake, the weakest association occurred in patients who reported a medium vitamin $\mathrm{E}$ intake, and a negative association was apparent in patients who reported a low vitamin E intake (Figure 1D). This suggests that not only did high vitamin E intake enhance the relationship between the relative abundance of Ruminococcus and IL-8 concentration, but also that the amount of vitamin E intake moderated this relationship in a highly sensitive manner.

$234 \quad$ Vitamin $\mathrm{C}$ intake had a significant main effect in the regression model involving the relationship 235 between the relative abundance of Ruminococcus and IL-8 concentrations, but there was no 236 "Ruminococcus $\times$ Vitamin C" interaction effect (Table S8). 
237

238

239

240

241

242

243

244

245

246

247

248

249

250

251

252

253

254

255

256

257

258

259

260

261

262

263

264

265

266

267

268

269

270

The relative abundance of Comamonas and cholesterol intake had significant effects on urinary IL-8. The "Comamonas $\times$ Cholesterol" interaction effect was also significant, and it explained $68.00 \%$ of the variance in IL-8 concentration (Table S9). The strongest positive association between the relative abundance of Comamonas and IL-8 concentration occurred in patients who reported a high cholesterol intake, and the weakest association occurred in patients who reported a low cholesterol intake (Figure 2A). This suggested that cholesterol intake enhanced the relationship between the relative abundance of Comamonas and IL-8.

Similarly, the relative abundance of Comamonas and magnesium intake had significant effects on urinary IL-8. The "Comamonas $\times$ Magnesium" interaction effect was also significant, and it explained $73.00 \%$ of the variance in IL-8 concentration (Table S10). The strongest positive association between the relative abundance of Comamonas and IL-8 concentration occurred in patients who reported a high magnesium intake, and the weakest association occurred in patients who reported a low magnesium intake (Figure 2B). This suggested that magnesium intake enhanced the relationship between Comamonas and IL-8 concentration.

\section{Discussion}

During the last decade, evidence has accumulated to support a role for the microbiota, including the bacterial communities in the gut and oral cavity, in T2DM patients (Navab-Moghadam et al., 2017; Larsen et al., 2010; Zhang et al., 2018). Not only do T2DM patients have a different microbiota profile from healthy subjects, but alterations in the microbiota are also responsible for immune responses, such as the expression of proinflammatory cytokines (Xiao et al., 2017; Pushpanathan et al., 2016). Both human and animal studies have shown that diet contributes to the composition of the gut microbiota (Egshatyan et al., 2015; Wen \& Duffy, 2017), and a gut microbiota signature that promotes intestinal inflammation may exist, thereby promoting the development of T2DM (Wen \& Duffy, 2017). Similar to previous findings relating to the gut and oral microbiota, our previous research demonstrated that T2DM patients have a different urinary microbiota composition from HCs, and the urinary microbiota composition was correlated with urinary IL-8 concentration in T2DM patients (Liu et al., 2017; Ling et al., 2017). In accordance with the "diet—gut microbiota—proinflammatory response" pathway described in previous work, we investigated whether diet moderates the relationship between the urinary microbiota and IL-8 concentration.

In this study, the relative abundance of Ruminococcus was positively associated with urinary IL-8. Ruminococcus spp. have rarely been reported in human urinary microbiota studies. A previous human urinary microbiome study demonstrated that Ruminococcus was positively associated with preterm birth (Ollberding et al., 2016), and it was detected in the urine of patients with chronic allograft dysfunction (Wu et al., 2018). These indicate that urinary Ruminococcus might be associated with the occurrence and 
271 development of some diseases. In our study, none of the subjects were diagnosed with UTI at recruitment 272 when their urine specimens were collected, but most samples having detectable Ruminococcus and IL-8. 273 This together with the positive correlation of Ruminococcus and urinary IL-8 level indicates that the 274 potential of members of Ruminococcus as a contributor of IL-8 and UTI in T2DM patients. Future studies 275 should investigate whether the UTI occurrence in the patients with detectable IL-8 is higher than those 276 with non-detectalbe IL-8.

277 Interestingly, water intake weakened the positive relationship between the relative abundance of 278 Ruminococcus and IL-8 concentration. Resolution of a UTI relies on the concentration of an antibiotic 279 within the urine, but highly concentrated urine reduced bactericidal activity against some pathogens 280 (Mikel \& Marta, 2003). In a recent randomely controlled trial, increased water intake was effective on 281 controlling the number of cystitis episodes in women with reccurrent cystitis (Hooton et al., 2018). 282 Meanwhile, increased water intake can reduce the dose of antimicrobial-regimens (Hooton et al., 2018).

Surprisingly, increasing fiber intake enhanced the relationship between the relative abundance of Ruminococcus and IL-8 concentration. In the gut, increased fiber intake improves the multiplication of Ruminococcus (Abell et al., 2008), and dietary fiber was inversely associated with inflammatory markers, including C-reactive protein (Wannamethee et al., 2009), interleukin-6 (Wannamethee et al., 2009; Qi et al., 2006), TNF- $\alpha-R 2$ (Qi et al., 2006; Ma et al., 2008). Therefore, it seems that fiber intake contribute

288

289

290

291

292

293

294

295

296

297

298

299

300

301

302

303 inversely to the relationship of Ruminococcus with inflammatory cytokines from the gut to the urine..

Both vitamin B3 and vitamin E intake exerted an enhancing effect on the positive relationship between Ruminococcus and IL-8 concentration. Since no previous studies reported that the roles of vitamin B3 and vitamine E on either Ruminoccocus growth and IL-8 expression, future studies should be focused on the role of the two vitamins in human urinary microbiome, then explore their moderation effects on the correlation between urinary microbiome and inflammatory markers.

Cholesterol intake had significant enhancing effects on the abundance of Comamonas and urinary IL-8. Comamonas is a bacterial genus in the phylum Proteobacteria. The current literatures supported that Comamonas spp. was related to infections including phlegmon, peritonitis, endocarditis, meningitis, septicemia, and UTI (Cooper et al., 2005; Biswas et al., 2014; Horowitz et al., 1990). Barua et al. found that nephritic syndrome patients with higher serum cholesterol were more susceptible to UTI than those with normal serum cholesterol, which might be because high cholesterol concentrations suppress lymphocyte function (Barua et al., 2016). With regard to magnesium, its intake had an enhancing effect on the relationship between the relative abundance of Comamonas and IL-8 concentration. However, it is not recommended that T2DM patients should restrict magnesium intake, since a previous in vitro study demonstrated that magnesium sulfate suppressed inflammatory response by human unbilical vein cells 
304 (Rochelson et al., 2007; Weglicki et al., 1992), and a previous rodent model study demonstrated that

305

306

307

308

309

310

311

312

313

314

315

316

317

318

319

320

321

322

323

324

325

326

327

328

329

330

331

332

333

334

335

336

337

338

339

340 magenesium-deficiency elevated circulating levels of inflammatory cytokines (Weglicki et al., 1992).

There were several limitations in our present study.Firslty,the participants were only from Zhejiang Province, China. It is known that different areas have different dietary patterns in China. For example, in Chinese southern areas (incuding Zhejiang province), the body sizes of the local population are generally small and short, and their diet is light and total intake is fewer than the northern areas. Thus, a multicenters study, including southern and northern areas in China, is needed to verify the results in the present study. Secondly, we have failed to detect the co-effect of bacteria on the correaltion between urinary microbiome and inflammatory reponse, since the 16S rDNA can only detect at the bacterial genus level and it is impossible to culture bacterial genus to examine their co-effect. Thirdly, T2DM patients might assess their dietary intake and exercise more accurately than the healthy subjects, because there were monthly healthcare education programs for patients in the communities in Zhejiang province. During the programs, the patients were instructed that dietary control and proper exercise could alleviate the progress of diabetes. Therefore, the patients might underestimate their dietary intake and overestimate their exercise. This might be one of the reasons why the patients in the present study had lower energy intake and equall exercise time than the healthy subjects, while their values of BMI were not different. Also, the response of assessment of dietary intake and and exercise might be different between the patients and healthy subjects, which might be a bias for assessing urinary microbiome and IL-8 production.

\section{Conclusions}

To our knowledge, this study is the first study to demonstrate the moderating effects of food intake on the relationship between the midstream urinary microbiota and urinary IL-8 concentration in humans. It is suggested that modulating the dietary patterns of patients based on urinary microbiota dysbiosis status and urinary IL-8 concentration might play a role in regulating inflammation response in urinary tract system.

\section{Acknowledgements}

We gratefully acknowledge the volunteers who participated in our study. We also thank the Charlesworth Group for editing the English in the manuscript.

\section{References}

Abell, G.C., Cooke, C.M., Bennett, C.N., Conlon, M.A., and McOrist, A.L. 2008. Phylotypes related to Ruminococcus bromii are abundant in the large bowel of humans and increase in response to a diet high in resistant starch. FEMS MICROBIOLOGY ECOLOGY 66:505-515. 10.1111/j.1574-6941.2008.00527.x

Barua, T., Sultana, R., Babul, F., Iqbal, S., Sharma, J., and Dutta, P. 2016. Urinary Tract Infection in Nephrotic Syndrome: A Hospital Based Cross-Sectional Study. MAA-O-SHISHU HOSPITAL MEDICAL COLLEGE JOURNAL 15:41-44.

Biswas, J.S., Fitchett, J., and O'Hara, G. 2014. Comamonas kerstersii and the perforated appendix. JOURNAL OF CLINICAL MICROBIOLOGY 52:3134. 10.1128/JCM.00909-14 
341

342

343

344

345

346

347

348

349

350

351

352

353

354

355

356

357

358

359

360

361

362

363

364

365

366

367

368

369

370

371

372

373

374

375

376

377

378

379

380

381

382

383

384

385

386

387

388

389

390

391

392

393

394

395

396

Carey, I., Critchley, J., DeWilde, S., Harris, T., Hosking, F., and Cook, D. 2018. Risk of Infection in Type 1 and Type 2 Diabetes Compared With the General Population: A Matched Cohort Study. DIABETES CARE 41:513-521. 10.2337/dc17-2131/-/DC1

Chavarro, J.E., Rosner, B.A., Sampson, L., Willey, C., Tocco, P., Willett, W.C., Chumlea, W.C., and Michels, K.B. 2009. Validity of adolescent diet recall 48 years later. AMERICAN JOURNAL OF EPIDEMIOLOGY 170:15631570. 10.1093/aje/kwp299

Cooper, G.R., Staples, E.D., Iczkowski, K.A., and Clancy, C.J. 2005. Comamonas (Pseudomonas) testosteroni endocarditis. CARDIOVASCULAR PATHOLOGY 14:145-149. 10.1016/j.carpath.2005.01.008

David, L.A., Maurice, C.F., Carmody, R.N., Gootenberg, D.B., Button, J.E., Wolfe, B.E., Ling, A.V., Devlin, A.S., Varma, Y., Fischbach, M.A., Biddinger, S.B., Dutton, R.J., and Turnbaugh, P.J. 2014. Diet rapidly and reproducibly alters the human gut microbiome. NATURE 505:559-563. 10.1038/nature12820

Egshatyan, L.V., Kashtanova, D.A., Popenko, A.S., Tkacheva, O.N., Tyakht, A.V., Alexeev, D.G., Karamnova, N.S., Kostryukova, E.S., Babenko, V.V., Vakhitova, M.T., and Boytsov, S.A. 2015. Gut microbiota and diet in patients with different glucose tolerance. ENDOCRINE CONNECTIONS 5:1-9. 10.1530/EC-15-0094

Fairchild, A.J., and MacKinnon, D.P. 2009. A General Model for Testing Mediation and Moderation Effects. PREVENTION SCIENCE 10:87-99. 10.1007/s11121-008-0109-6

Hirji, I., Guo, Z., Andersson, S.W., Hammar, N., and Gomez-Caminero, A. 2012. Incidence of urinary tract infection among patients with type 2 diabetes in the UK General Practice Research Database (GPRD). JOURNAL OF DIABETES AND ITS COMPLICATIONS 26:513-516. 10.1016/j.jdiacomp.2012.06.008

Hooton, T.M., Vecchio, M., Iroz, A., Tack, I., Dornic, Q., Seksek, I., and Lotan, Y. 2018. Effect of Increased Daily Water Intake in Premenopausal Women With Recurrent Urinary Tract Infections. INTERNAL MEDICINE 178:1509. 10.1001/jamainternmed.2018.4204

Horowitz, H., Gilroy, S., Feinstein, S., and Gilardi, G. 1990. Endocarditis associated with Comamonas acidovorans. JOURNAL OF CLINICAL MICROBIOLOGY 28:143-145.

Larsen, N., Vogensen, F.K., van den Berg, F.W., Nielsen, D.S., Andreasen, A.S., Pedersen, B.K., Al-Soud, W.A., Sorensen, S.J., Hansen, L.H., and Jakobsen, M. 2010. Gut microbiota in human adults with type 2 diabetes differs from non-diabetic adults. PLOS ONE 5:e9085. 10.1371/journal.pone.0009085

Ling, Z., Liu, F., Shao, L., Cheng, Y., and Li, L. 2017. Dysbiosis of the Urinary Microbiota Associated With Urine Levels of Proinflammatory Chemokine Interleukin-8 in Female Type 2 Diabetic Patients. FRONTIERS IN IMMUNOLOGY 8. 10.3389/fimmu.2017.01032

Liu, F., Ling, Z., Xiao, Y., Lv, L., Yang, Q., Wang, B., Lu, H., Zheng, L., Jiang, P., Wang, W., and Li, L. 2017. Dysbiosis of urinary microbiota is positively correlated with type 2 diabetes mellitus. ONCOTARGET 8:3798-3810. 10.18632/oncotarget. 14028

Ma, Y., Hébert, J.R., Li, W., Bertone-Johnson, E.R., Olendzki, B., Pagoto, S.L., Tinker, L., Rosal, M.C., Ockene, I.S., Ockene, J.K., Griffith, J.A., and Liu, S. 2008. Association between dietary fiber and markers of systemic inflammation in the Women's Health Initiative Observational Study. NUTRITION 24:941-949. 10.1016/j.nut.2008.04.005

Mandal, S., Godfrey, K.M., McDonald, D., Treuren, W.V., Bjornholt, J.V., Midtvedt, T., Moen, B., Rudi, K., Knight, R., Brantsaeter, A.L., Peddada, S.D., and Eggesbo, M. 2016. Fat and vitamin intakes during pregnancy have stronger relations with a pro-inflammatory maternal microbiota than does carbohydrate intake. MICROBIOME 4:55. 10.1186/s40168-016-0200-3

Mikel, G., and Marta, K. 2003. Does Fluid Intake Influence the Risk for Urinary Incontinence, Urinary Tract Infection, and Bladder Cancer. J WOCN 30:126-131. 10.1067/mjw.2003

Navab-Moghadam, F., Sedighi, M., Khamseh, M.E., Alaei-Shahmiri, F., Talebi, M., Razavi, S., and Amirmozafari, N. 2017. The association of type II diabetes with gut microbiota composition. MICROBIAL PATHOGENESIS 110:630-636. 10.1016/j.micpath.2017.07.034

Nichols, G.A., Brodovicz, K.G., Kimes, T.M., Déruaz-Luyet, A., and Bartels, D.B. 2017. Prevalence and incidence of urinary tract and genital infections among patients with and without type 2 diabetes. J DIABETES COMPLICATIONS 31:1587-1591. 10.1016/j.jdiacomp.2017.07.018

Ollberding, N.J., Volgyi, E., Macaluso, M., Kumar, R., Morrow, C., Tylavsky, F.A., and Piyathilake, C.J. 2016. Urinary Microbiota Associated with Preterm Birth: Results from the Conditions Affecting Neurocognitive Development and Learning in Early Childhood (CANDLE) Study. PLOS ONE 11:e162302. 10.1371/journal.pone.0162302

Price, T.K., Dune, T., Hilt, E.E., Thomas-White, K.J., Kliethermes, S., Brincat, C., Brubaker, L., Wolfe, A.J., Mueller, E.R., and Schreckenberger, P.C. 2016. The Clinical Urine Culture: Enhanced Techniques Improve Detection of Clinically Relevant Microorganisms. JOURNAL OF CLINICAL MICROBIOLOGY 54:1216-1222.

Peerf reviewing PDF | (2019:06:38336:2:0:NEW 17 Dec 2019) 


\subsection{8/JCM.00044-16}

Pushpanathan, P., Srikanth, P., Seshadri, K., Selvarajan, S., Pitani, R., Kumar, T., and Janarthanan, R. 2016. Gut Microbiota in Type 2 Diabetes Individuals and Correlation with Monocyte Chemoattractant Protein1 and Interferon Gamma from Patients Attending a Tertiary Care Centre in Chennai, India. INDIAN JOURNAL OF ENDOCRINOLOGY AND METABOLISM 20:523. 10.4103/2230-8210.183474

Qi, L., Rob, M.V.D., Liu, S., Franz, M., Mantzoros, C., and Hu, F.B. 2006. Whole-Grain, Bran, and Cereal Fiber Intakes and Markers of Systemic Inflammation in Diabetic Women. DIABETES CARE 29:207-211. 10.2337/diacare.29.02.06.dc05-1903

Rao, W.H., Evans, G.S., and Finn, A. 2001. The significance of interleukin 8 in urine. ARCHIVES OF DISEASE IN CHILDHOOD 85:256-262.

Ribeiro, A.A., Azcarate-Peril, M.A., Cadenas, M.B., Butz, N., Paster, B.J., Chen, T., Bair, E., and Arnold, R.R. 2017. The oral bacterial microbiome of occlusal surfaces in children and its association with diet and caries. PLOS ONE 12:e180621. 10.1371/journal.pone.0180621

Rochelson, B., Dowling, O., Schwartz, N., and Metz, C.N. 2007. Magnesium sulfate suppresses inflammatory responses by human umbilical vein endothelial cells (HuVECs) through the NF к B pathway. JOURNAL OF REPRODUCTIVE IMMUNOLOGY 73:101-107. 10.1016/j.jri.2006.06.004

Seksik, P., Rigottier-Gois, L., Gramet, G., Sutren, M., Pochart, P., Marteau, P., Jian, R., and Dore, J. 2003. Alterations of the dominant faecal bacterial groups in patients with Crohn's disease of the colon. GUT 52:237-242. 10.1136/gut.52.2.237

Shields-Cutler, R.R., Crowley, J.R., Hung, C.S., Stapleton, A.E., Aldrich, C.C., Marschall, J., and Henderson, J.P. 2015. Human Urinary Composition Controls Antibacterial Activity of Siderocalin. JOURNAL OF BIOLOGICAL CHEMISTRY 290:15949-15960. 10.1074/jbc.M115.645812

Simpson, H.L., and Campbell, B.J. 2015. Dietary fibre-microbiota interactions. ALIMENTARY PHARMACOLOGY AND THERAPEUTICS 42:158-179. 10.1111/apt.13248

Singh, R.K., Chang, H.W., Yan, D., Lee, K.M., Ucmak, D., Wong, K., Abrouk, M., Farahnik, B., Nakamura, M., Zhu, T.H., Bhutani, T., and Liao, W. 2017. Influence of diet on the gut microbiome and implications for human health. JOURNAL OF TRANSLATIONAL MEDICINE 15:73. 10.1186/s12967-017-1175-y

Tai, N., Wong, F.S., and Wen, L. 2015. The role of gut microbiota in the development of type 1, type 2 diabetes mellitus and obesity. REVIEWS IN ENDOCRINE AND METABOLIC DISORDERS 16:55-65. 10.1007/s11154-0159309-0

Thomas-White, K., Forster, S.C., Kumar, N., Van Kuiken, M., Putonti, C., Stares, M.D., Hilt, E.E., Price, T.K., Wolfe, A.J., and Lawley, T.D. 2018. Culturing of female bladder bacteria reveals an interconnected urogenital microbiota. NATURE COMMUNICATIONS 9:1557. 10.1038/s41467-018-03968-5

Tilg, H., and Moschen, A.R. 2015. Food, Immunity, and the Microbiome. GASTROENTEROLOGY 148:1107-1119. 10.1053/j.gastro.2014.12.036

Walker, A.W., Ince, J., Duncan, S.H., Webster, L.M., Holtrop, G., Ze, X., Brown, D., Stares, M.D., Scott, P., Bergerat, A., Louis, P., McIntosh, F., Johnstone, A.M., Lobley, G.E., Parkhill, J., and Flint, H.J. 2011. Dominant and diet-responsive groups of bacteria within the human colonic microbiota. ISME JOURNAL 5:220-230. 10.1038/ismej.2010.118

Wang, Y., Luo, X., Mao, X., Tao, Y., Ran, X., Zhao, H., Xiong, J., and Li, L. 2017. Gut microbiome analysis of type 2 diabetic patients from the Chinese minority ethnic groups the Uygurs and Kazaks. PLOS ONE 12:e172774. 10.1371/journal.pone.0172774

Wannamethee, S.G., Whincup, P.H., Thomas, M.C., and Sattar, N. 2009. Associations Between Dietary Fiber and Inflammation, Hepatic Function, and Risk of Type 2 Diabetes in Older Men: Potential mechanisms for the benefits of fiber on diabetes risk. DIABETES CARE 32:1823-1825. 10.2337/dc09-0477

Weglicki, W.B., Phillips, T.M., Freedman, A.M., Cassidy, M.M., and Dickens, B.F. 1992. Magnesium-deficiency elevates circulating levels of inflammatory cytokines and endothelin. MOLECULAR AND CELLULAR BIOCHEMISTRY 110:169-173. 10.1007/BF02454195

Wen, L., and Duffy, A. 2017. Factors Influencing the Gut Microbiota, Inflammation, and Type 2 Diabetes. JOURNAL OF NUTRITION 147:1468S-1475S. 10.3945/jn.116.240754

Wilke, T., Boettger, B., Berg, B., Groth, A., Mueller, S., Botteman, M., Yu, S., Fuchs, A., and Maywald, U. 2015. Epidemiology of urinary tract infections in type 2 diabetes mellitus patients: An analysis based on a large sample of 456,586 German T2DM patients. JOURNAL OF DIABETES AND ITS COMPLICATIONS 29:1015-1023. 10.1016/j.jdiacomp.2015.08.021

Wu, J.F., Muthusamy, A., Al-Ghalith, G.A., Knights, D., Guo, B., Wu, B., Remmel, R.P., Schladt, D.P., Alegre, M., Oetting, W.S., Jacobson, P.A., and Israni, A.K. 2018. Urinary microbiome associated with chronic allograft 
453 dysfunction in kidney transplant recipients. CLINICAL TRANSPLANTATION 32:e13436. 10.1111/ctr.13436

454 Xiao, E., Mattos, M., Vieira, G.H.A., Chen, S., Corrêa, J.D., Wu, Y., Albiero, M.L., Bittinger, K., and Graves, D.T. 455 2017. Diabetes Enhances IL-17 Expression and Alters the Oral Microbiome to Increase Its Pathogenicity. $C E L L$ 456 HOST \& MICROBE 22:120-128. 10.1016/j.chom.2017.06.014

457 Zaki, M.S. 2008. Interleukin 8 is a surrogate marker for rapid diagnosis of bacteriuria. IMMUNOLOGICAL 458 INVESTIGATIONS 37:694-703. 10.1080/08820130802307278

459 Zhang, Y., Wang, X., Li, H., Ni, C., Du, Z., and Yan, F. 2018. Human oral microbiota and its modulation for oral 460 health. BIOMEDICINE \& PHARMACOTHERAPY 99:883-893. 10.1016/j.biopha.2018.01.146

461 Zhao, W., Huang, Z., Zhang, X., He, L., Willett, W., Wang, J., Hasegawa, K., and Chen, J. 2010. Reproducibility 462 and Validity of a Chinese Food Frequency Questionnaire. BIOMEDICAL AND ENVIRONMENTAL SCIENCES 463 23:1-38. https://doi.org/10.1016/S0895-3988(11)60014-7

464 Zheng, Y., Ley, S.H., and Hu, F.B. 2018. Global aetiology and epidemiology of type 2 diabetes mellitus and its 465 
Figure 1

\section{Diet, Ruminococcus and IL-8}

Figure 1

A

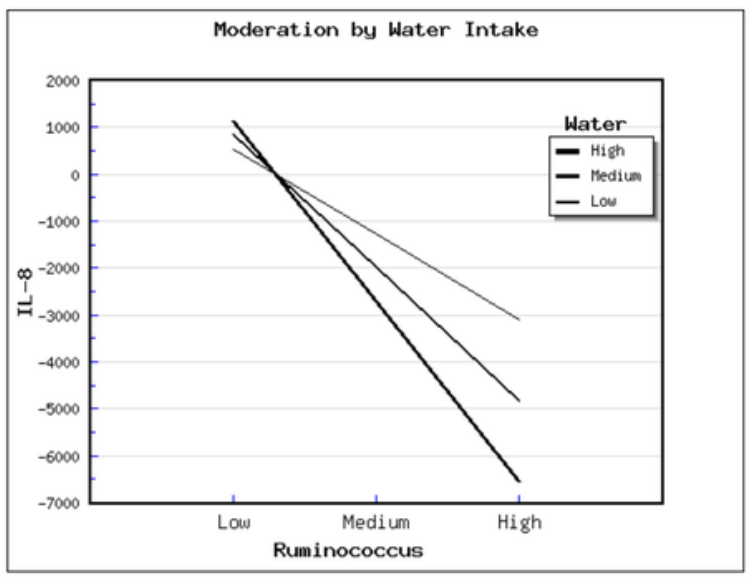

C

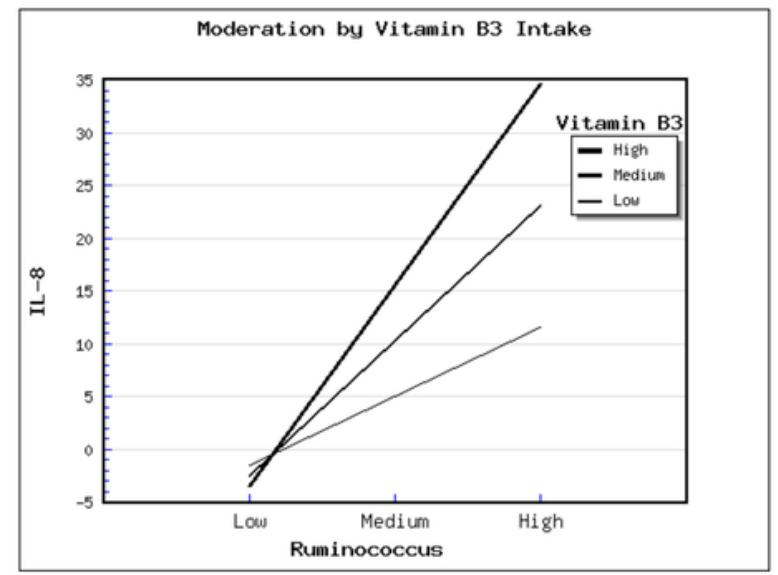

B

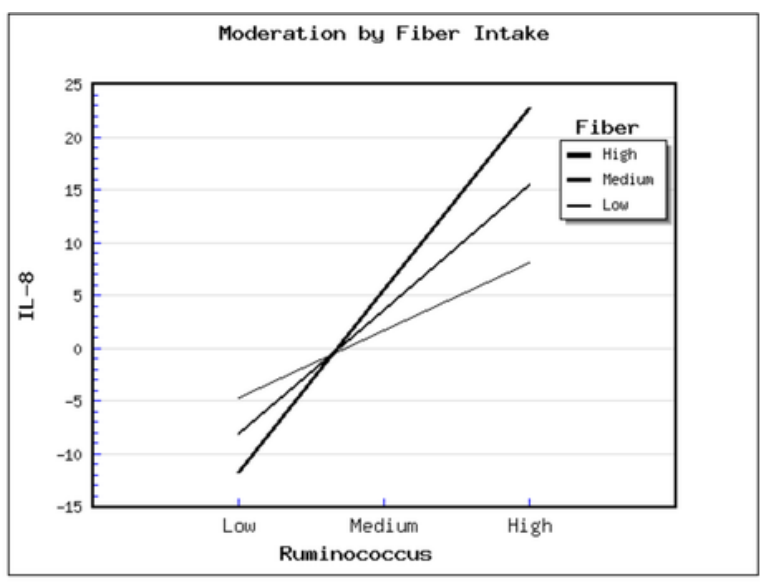

D

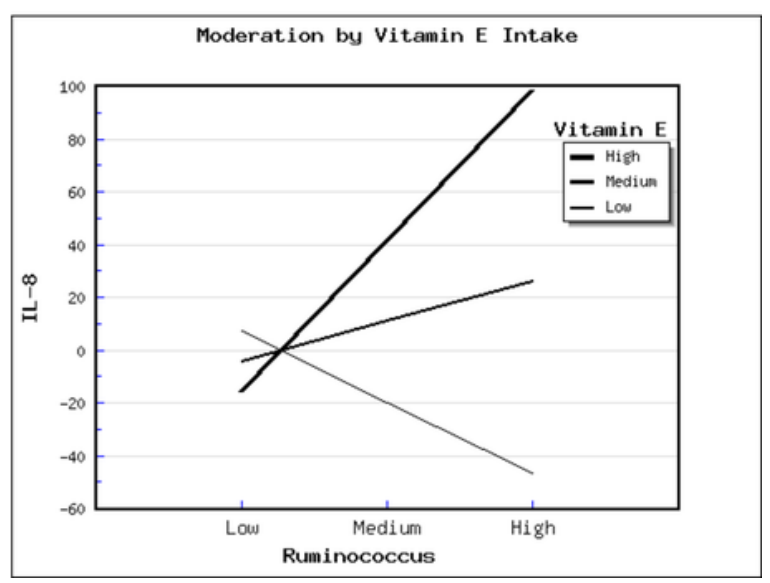


Figure 2

\section{Diet, Comamomas and IL-8}

Figure 2

A

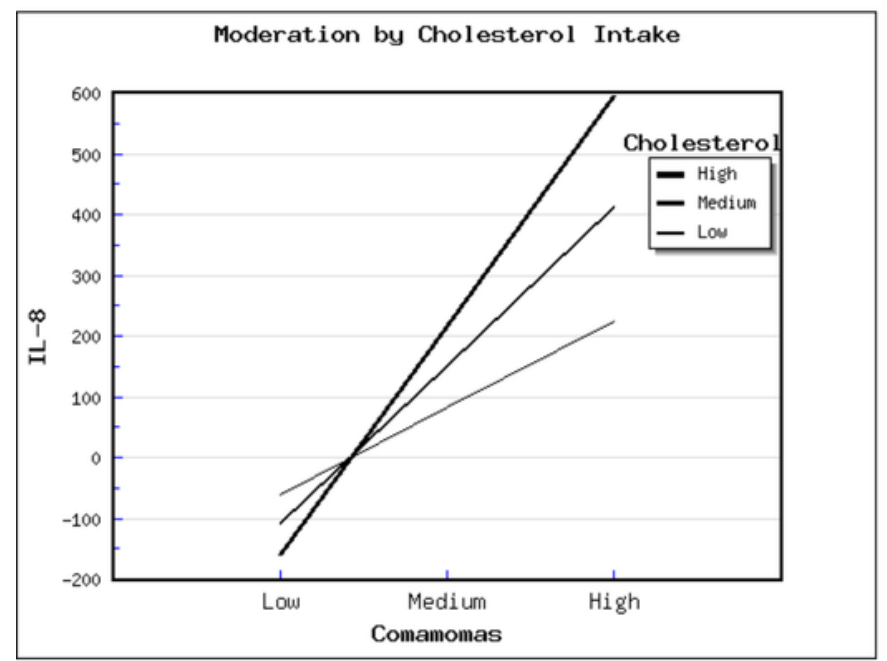

B

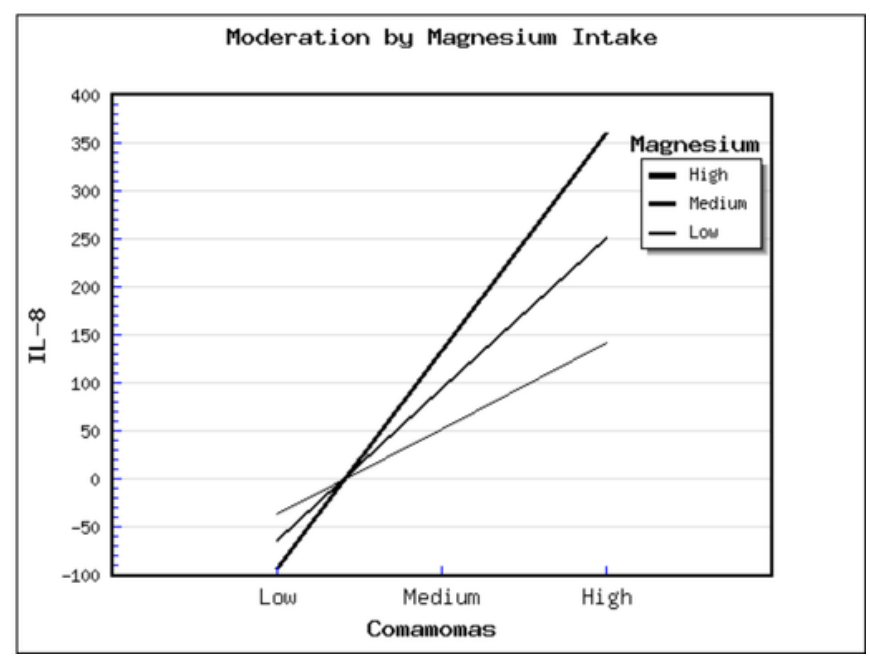


Table $\mathbf{1}$ (on next page)

Characteristics of T2DM subjects

Table 1 
Table 2 (on next page)

Comparison of nutrient intake between T2DM and HCs

Table 2 
Table 2 Comparison of nutrient intake between T2DM and HCs

\begin{tabular}{|c|c|c|c|}
\hline Nutrients & T2DM & $\mathrm{HCs}$ & p-value \\
\hline Water $(\mathrm{mL} / \mathrm{d})$ & $2526.04 \pm 903.79$ & $2484.24 \pm 829.94$ & 0.79 \\
\hline Energy（kcal / d ） & $1256.55 \pm 479.72$ & $1469.07 \pm 626.63$ & 0.03 \\
\hline Protein $(\mathrm{g} / \mathrm{d})$ & $60.73 \pm 27.29$ & $72.85 \pm 46.09$ & 0.06 \\
\hline Fat $(\mathrm{g} / \mathrm{d})$ & $57.75 \pm 30.05$ & $59.32 \pm 29.40$ & 0.76 \\
\hline SFA $(\% \mathrm{TE} / \mathrm{d})$ & $11.65 \pm 6.39$ & $13.73 \pm 11.29$ & 0.18 \\
\hline MUFA (\% TE / d) & $18.67 \pm 11.96$ & $19.15 \pm 11.32$ & 0.81 \\
\hline PUFA (\% TE / d) & $16.71 \pm 10.68$ & $15.10 \pm 8.68$ & 0.33 \\
\hline Carbohydrate $(\mathrm{g} / \mathrm{d})$ & $151.46 \pm 58.59$ & $184.38 \pm 94.23$ & 0.01 \\
\hline Fiber $(\mathrm{g} / \mathrm{d})$ & $9.26 \pm 4.54$ & $12.50 \pm 17.20$ & 0.13 \\
\hline Cholesterol $(\mathrm{mg} / \mathrm{d})$ & $472.36 \pm 212.18$ & $486.47 \pm 179.68$ & 0.67 \\
\hline Vit A $(\mu \mathrm{gRE} / \mathrm{d})$ & $745.95 \pm 315.96$ & $1032.52 \pm 1351.94$ & 0.09 \\
\hline Retinol $(\mu \mathrm{g} / \mathrm{d})$ & $265.07 \pm 178.10$ & $380.30 \pm 632.97$ & 0.15 \\
\hline Vit B1 $(\mathrm{mg} / \mathrm{d})$ & $0.70 \pm 0.32$ & $0.77 \pm 0.32$ & 0.18 \\
\hline Vit B2（mg / d ） & $0.88 \pm 0.33$ & $1.18 \pm 0.93$ & 0.01 \\
\hline Vit B3（mg / d ） & $13.92 \pm 6.84$ & $18.77 \pm 15.09$ & 0.02 \\
\hline Vit C ( $\mathrm{mg} / \mathrm{d})$ & $107.44 \pm 40.45$ & $127.10 \pm 62.21$ & 0.03 \\
\hline Vit E $(\mathrm{mg} / \mathrm{d})$ & $13.83 \pm 42.49$ & $7.61 \pm 20.65$ & 0.27 \\
\hline Calcium $(\mathrm{mg} / \mathrm{d})$ & $687.33 \pm 323.02$ & $831.39 \pm 681.37$ & 0.11 \\
\hline Phosphorus ( $\mathrm{mg} / \mathrm{d})$ & $936.87 \pm 388.41$ & $1090.99 \pm 547.12$ & 0.06 \\
\hline Potassium $(\mathrm{mg} / \mathrm{d})$ & $1704.71 \pm 678.50$ & $2262.05 \pm 1882.30$ & 0.02 \\
\hline Sodium $(\mathrm{mg} / \mathrm{d})$ & $2859.29 \pm 1586.14$ & $2788.47 \pm 1585.96$ & 0.79 \\
\hline Magnesium $(\mathrm{mg} / \mathrm{d})$ & $267.73 \pm 116.61$ & $325.39 \pm 233.91$ & 0.07 \\
\hline Iron $(\mathrm{mg} / \mathrm{d})$ & $21.47 \pm 10.75$ & $26.91 \pm 24.19$ & 0.09 \\
\hline Zinc $(\mathrm{mg} / \mathrm{d})$ & $9.49 \pm 3.84$ & $11.47 \pm 6.54$ & 0.03 \\
\hline Selenium $(\mu \mathrm{g} / \mathrm{d})$ & $38.42 \pm 17.68$ & $49.71 \pm 29.51$ & 0.01 \\
\hline Copper $(\mathrm{mg} / \mathrm{d})$ & $1.78 \pm 1.00$ & $2.32 \pm 2.47$ & 0.10 \\
\hline Manganese $(\mathrm{mg} / \mathrm{d})$ & $4.17 \pm 1.80$ & $8.95 \pm 32.13$ & 0.23 \\
\hline
\end{tabular}

Abbreviations: TE: total energy intake; RE: retinol equivalent; SFA: saturated fatty acid; MUFA: monounsaturated fatty acid; 